\title{
Emociones, estilos de afrontamiento y agresividad en la adolescencia
}

\section{Emotions, Coping Style and Aggression during Adolescence}

Recibido: diciembre 23 de 2011 | Revisado: febrero 17 de 2012 | Aceptado: febrero 27 de 2012

\author{
ViCENTA MESTRE* \\ PAUla SAMPER \\ Ana María Tur-Porcar \\ Universidad de Valencia, España \\ MARÍA CRISTINA RichaUd DE MinZI** \\ BELÉN MESURADO \\ Centro Interdisciplinario de Investigaciones en Psicología \\ Matemática y Experimental, Buenos Aires, Argentina
}

SICI: 2011-2277(201212)11:4<1263:EEAAEA >2.0.CO;2-5

Para citar este artículo: Mestre, V., Samper, P., Tur-Porcar, A. M., Richaud de Minzi, M. C. \& Mesurado, B. (2012). Emociones, estilos de afrontamiento y agresividad en la adolescencia. Universitas Psychologica, 11(4), 1263-1275.

* Departamento de Psicología Básica. Facultad de Psicología. Avda. Blasco Ibáñez, 21. 46010. Valencia, España.E-mails: Maria.V.Mestre@uv.es, Paula. Samper@uv.es, Ana.Tur@uv.es. ResearcherID: Mestre, V. A-8102-2013; Samper, P. A-6854-2013.

** Centro Interdisciplinario de Investigaciones en Psicología Matemática y Experimental (CIIPME), dependiente del Consejo Nacional de Investigaciones Científicas y Técnicas (CONICET). Tte. Gral. Perón 2158 - 1040, Buenos Aires, Argentina. Emails:minzi@ciudad.com.ar, mesuradob@gmail. com

\section{RES UMEN}

Este estudio analiza la relación entre las estrategias de afrontamiento y las emociones para determinar en qué medida son procesos relacionados con la conducta agresiva. Se parte del supuesto de que en la agresividad influyen los mecanismos de afrontamiento en la resolución de problemas y el manejo de las emociones: inestabilidad emocional (falta de autocontrol ante situaciones que producen tensión) o empatía (sentimientos orientados al otro que tiene un problema o necesidad). Se ha evaluado una muestra de 1.557 niños y niñas, con un rango de edad entre 12 y 15 años, escolarizados en primer ciclo de Educación Secundaria Obligatoria (ESO) en 36 Centros escolares de la Comunidad Valenciana, seleccionados aleatoriamente. Los resultados indican claras diferencias entre los sujetos con alta y baja agresividad y los mecanismos de afrontamiento que utilizan, los adolescentes más agresivos utilizan en mayor medida un afrontamiento improductivo, mientras que los menos agresivos aplican estrategias más centradas en la resolución del problema. En todos los modelos de ecuaciones estructurales, el efecto directo de la inestabilidad emocional y la empatía sobre la agresividad es significativa, siempre positivo en el primer caso pero negativo en el segundo. Además, la empatía favorece el afrontamiento centrado en la resolución del problema, mientras que la inestabilidad emocional se relaciona positivamente con el afrontamiento improductivo y este con la agresividad.

Palabras clave autores

Emociones, estilos de afrontamiento, agresividad, adolescencia.

Palabras clave descriptores

Resolución de problemas, Psicología del Desarrollo, investigación cuantitativa.

\begin{abstract}
A B S T R A C T
This study assesses the relation between coping strategies and emotions to know to what extend these are processes related to aggressive behavior. We assume that the aggression influence coping mechanisms in solving problems and handling of emotions: emotional instability (lack of self-control in stressful situations) or empathy (feelings faced to "other" who has a problem or need). A sample of 1.557 boys and girls, with an age range of $12-15$ years, enrolled in first-cycle of Compulsory Secondary Education in 36 Scholar Centers in the Valencian Community, randomly selected, was evaluated. The results indicate clear differences between high and low aggression and coping mechanisms that they use. More aggressive adolescents used to a
\end{abstract}


greater extent an unproductive coping, while less aggressive adolescents apply strategies more focused on solving the problem. In all structural equation models, the direct effect of emotional instability and empathy on aggression is significant, always positive in first case but negative in the second one. Besides, empathy promotes coping centered on the resolution of the problem whereas emotional instability is positively related to unproductive coping and this one with aggression.

Key words authors

Emotions, Coping Style, Aggression, Adolescence.

Key words plus

Resolution of problems, Development Psychology, Quantitative Research.

\section{Introducción}

En los últimos años se ha producido un incremento de violencia y agresiones en diferentes contextos. La agresividad ha aumentado en el ámbito familiar y escolar, y en dichas conductas están asumiendo un protagonismo creciente los adolescentes. Diferentes estudios concluyen un aumento de la conducta agresiva y de la inestabilidad emocional desde los últimos años de la infancia hasta la adolescencia (Chaux, 2003; Farrington, 2004; Samper, Aparici $\&$ Mestre, 2006).

La agresividad, en sus diferentes manifestaciones verbal, física y fría o más sutil, la ira y la inestabilidad emocional, estimulan las conductas antisociales. Todas ellas, una vez activadas, alimentan y sostienen la conducta más allá del control voluntario de la persona (Mestre, Tur, Samper \& Latorre, 2010). La inestabilidad emocional constituye un factor de riesgo que se relaciona positivamente con la agresividad física y verbal, y negativamente con la disposición a establecer relaciones empáticas y prosociales con el entorno (Del Barrio, Moreno \& López, 2001; Tur, Mestre \& Del Barrio, 2004). Además, la agresividad y la inestabilidad emocional son dos variables que correlacionan fuertemente de manera positiva y significativa entre sí (Carlo, Mestre, Samper, Tur \& Armenta, 2010; Del Barrio, Carrasco, Rodríguez \& Gordillo, 2009; Mestre et al., 2010; Tur et al., 2004).

Aunque hasta fechas recientes el estudio de la agresividad y la violencia se ha centrado más en los aspectos negativos y en los factores de riesgo, que facilitaban el desarrollo de tales conductas desadaptadas, en los últimos años está cobrando protagonismo un enfoque más proactivo, que entronca en los principios de la psicología positiva en la línea definida por Seligman y Csikszentmihalyi (2000). Desde esta perspectiva, el interés se centra en el análisis de los factores que inciden en el desarrollo de la personalidad ajustada (personal y socialmente), y por tanto en el fortalecimiento de los factores de protección en los adolescentes que inhiben la conducta agresiva.

Entre los factores personales de protección de la agresividad destaca la empatía, entendida como la capacidad de ponerse en el lugar del otro, demostrar preocupación por el otro, comprender al otro y, además, mantener conductas de acercamiento hacia el otro, especialmente como un factor de protección de la agresividad (Carlo et al., 2010; Garaigordobil, 2009a; Mestre, Samper \& Frías, 2002; Vaish, Carpenter \& Tomasello, 2009) y como un proceso que favorece la adaptación e integración social (Caprara, Barbanelli, Pastorelli, Bandura \& Zimargdo, 2000; Garaigordobil, 2009b), así como las habilidades sociales que facilitan la relación con los iguales (Dovidio \& Penner, 2004; Mestre, Samper \& Frías, 2004).

Estudios recientes constatan la relación entre inteligencia emocional y relaciones interpersonales, en concreto entre inteligencia emocional, empatía y autocontrol en las situaciones sociales y las relaciones afectivas. Las personas más estables emocionalmente poseen una mayor autoestima, más capacidad de autocontrol en situaciones adversas, en definitiva tienden a la planificación de la acción, actuando de forma eficaz y al afrontamiento de situaciones, aprendiendo de estas aun cuando suponen fracaso (Bermúdez, Teva \& Sánchez, 2003), por el contrario los sujetos impulsivos presentarían dificultades para resolver tareas por la precipitación de respuestas, un menor control atencional y un uso inadecuado de estrategias de tipo analítico. De esta manera, la impulsividad cognitiva se relaciona con otros procesos cognitivos como el afrontamiento y la resolución de problemas (Arán-Filippetti \& Richaud, 2011). En población infantil existen resultados que relacionan las conductas disruptivas 
con dificultades para autorregular las emociones y con mecanismos de afrontamiento inadecuados, que impiden conductas alternativas a la agresión (Ison-Zintilini \& Morelato, 2008). En definitiva, el desarrollo de la capacidad para ponerse en el lugar del otro integra procesos cognitivos y emocionales implicados en la disposición prosocial y que contribuyen a una conducta prosocial más autónoma y madura como contrapuesta a la conducta agresiva.

Por otra parte, los mecanismos de afrontamiento son procesos que pueden regular o canalizar las emociones e influir en las respuestas más o menos adaptadas a la situación o problema que la persona vive en un momento dado. El afrontamiento implica siempre la adquisición de un cierto control de la situación, que puede oscilar entre un dominio total y un mero control de las emociones suscitadas por el evento estresante. La mayoría de los estudios sobre afrontamiento (Páez, 1993; Perona \& Galán, 2001; Richaud, 2006) coinciden en determinar tres dominios de las estrategias según estén dirigidas a la valoración (afrontamiento cognitivo: intento de encontrar significado al suceso y valorarlo de manera que resulte menos desagradable), dirigidas al problema (afrontamiento conductual: conducta dirigida a confrontar la realidad, manejando sus consecuencias) y a la emoción (afrontamiento afectivo: regulación de los aspectos emocionales e intento de mantener el equilibrio afectivo). Los estilos de afrontamiento son especialmente importantes a medida que se llega a la adolescencia, momento en que la importancia del grupo y la necesidad de autoafirmación hacen imprescindibles las habilidades de afrontamiento y la resolución no violenta de los conflictos. El afrontamiento desempeña un papel mediador importante entre las experiencias estresantes a las que están sometidas las personas, los recursos personales y sociales con los que cuentan para hacerles frente y las consecuencias que se derivan (Bermúdez, Teva \& Buela-Casal, 2009; Gómez-Fraguela, Luengo, Romero, Villar \& Sobral, 2006).

Desde este marco teórico se presenta la investigación, orientada a detectar los mecanismos de afrontamiento que mayormente utilizan los chicos y chicas al final de la infancia y primeros años de la adolescencia, y en qué medida dichas estrategias interactúan con la emocionalidad positiva y negativa para predecir la conducta agresiva. El objetivo general es analizar la relación entre las estrategias de afrontamiento y la gestión de las emociones, para determinar en qué medida influyen sobre la conducta agresiva, identificando para ello mecanismos de afrontamiento relacionados con emociones positivas (empatía) y con emociones negativas (inestabilidad emocional) y su función en la agresividad. Se parte del supuesto de que la agresividad va a estar relacionada con los mecanismos de afrontamiento y el manejo de las emociones: inestabilidad emocional (falta de autocontrol ante situaciones que producen tensión) o empatía (sentimientos orientados al otro que tiene un problema o una necesidad). Se formulan las siguientes hipótesis:

1. Las estrategias de afrontamiento centradas en la resolución del problema y en la relación con los demás guardan relación con menos agresividad, mientras que un estilo de afrontamiento improductivo se relaciona con una agresividad mayor.

2. Al mismo tiempo, la empatía y la inestabilidad emocional tienen un efecto directo sobre la agresividad.

3. La empatía y la inestabilidad emocional en la niñez tardía y en la adolescencia temprana tiene un efecto diferencial según los diferentes tipos de afrontamiento (improductivo, resolución de problemas y relación con los demás).

La tercera hipótesis da origen a tres modelos teóricos de acuerdo al tipo de afrontamiento analizado, en cada grupo de edad (niñez tardía y adolescencia temprana), bajo el supuesto de que la edad tiene una influencia sobre la forma en que estas variables se relacionan.

\section{Método}

\section{Participantes}

La muestra estuvo compuesta por 1.557 adolescentes, 818 hombres (52.6\%) y 738 mujeres (47.4\%), con un rango de edad entre 12 y 15 años, escolarizados en primer ciclo de Educación Secundaria 
Obligatoria. Todos ellos pertenecen a 36 centros educativos seleccionados aleatoriamente de diferentes centros educativos, que atienden alumnos que presentan dificultades de inserción escolar por encontrarse en posición desfavorable, derivada de situaciones sociales, culturales, económicas, étnicas o personales. De este modo, en la muestra seleccionada están representados todos los colectivos escolarizados en la población.

\section{Instrumentos}

Escala de Agresividad Física y Verbal [AFV] (Caprara \& Pastorelli, 1993; Del Barrio et al., 2001)

Se trata de una escala de 20 ítems que evalúa la conducta de hacer daño a otros física y verbalmente. El formato de respuesta es de tres alternativas ( $a$ menudo, algunas veces o nunca), según la frecuencia de aparición de la conducta. El índice de fiabilidad obtenido con la muestra objeto de estudio es de 0.8 .

Escala de Inestabilidad Emocional [IE] (Caprara \& Pastorelli, 1993; Del Barrio et al., 2001)

Describe la conducta que indica una falta de autocontrol en situaciones sociales como resultado de la escasa capacidad para frenar la impulsividad y la emocionalidad. Incluye 20 ítems con tres alternativas de respuesta (a menudo, algunas veces o nunca). El índice de fiabilidad obtenido con la muestra objeto de estudio es de 0.87 .

Índice de empatía para niños y adolescentes (Bryant, 1982; Mestre, PérezDelgado, Frías \& Samper, 1999)

Este instrumento es una medida del componente emocional de la empatía. Consta de 22 ítems. El formato de respuesta es dicotómico (sí/no), puntuando la respuesta que contribuye a una actitud empática. El análisis de la consistencia interna del instrumento llevado a cabo por Bryant (1982) señala un alfa medio de Cronbach de 0.67, aumentando con la edad de los sujetos. Los análisis de fiabilidad en nuestra muestra indican un alfa de Cronbach de 0.64. En otros estudios se han obtenido índices similares (Mestre et al., 1999).

Escala de Afrontamiento para Adolescentes (Frydenberg Eु Lewis, 1995; Pereña \& Seisdedos, 1997)

Evalúa cómo el joven entre 12 y 18 años afronta sus problemas en general. Concretamente, se diferencian 18 estrategias de afrontamiento que pueden agruparse en tres estilos básicos de afrontamiento: 1) Dirigido a la resolución del problema, reúne una serie de estrategias dirigidas a modificar la situación problemática para hacerla menos estresante, tales como: buscar diversiones relajantes, distracción física, esforzarse y tener éxito, fijarse en lo positivo y concentrarse en resolver el problema; 2) Afrontamiento en relación con los demás, reúne estrategias como: buscar apoyo espiritual, invertir en amigos íntimos, buscar ayuda profesional, buscar apoyo social, buscar pertenencia y acción social; 3) Afrontamiento improductivo, concentra estrategias que tienen que ver con: autoinculparse, hacerse ilusiones, ignorar el problema, falta de afrontamiento, preocuparse, reservarlo para sí y reducción de la tensión. El análisis de la consistencia interna del instrumento señala un alfa medio de Cronbach de 0.75 .

\section{Procedimiento}

La aplicación de los instrumentos se realizó de forma colectiva y en horas lectivas, en cada aula y para cada uno de los niveles de edad y estudios analizados. Las instrucciones para contestar los cuestionarios se explicaron oralmente antes de la cumplimentación. El pase de las pruebas se llevó a cabo en una sesión de 50 minutos a una hora aproximadamente, en las diferentes aulas seleccionadas aleatoriamente. Previamente a la aplicación de los instrumentos de evaluación se informó a los directores de los centros escolares seleccionados para participar en la investigación, a los profesores y a las familias, con el objetivo de pedir su colaboración y garantizar el anonimato de los datos. El diseño de 
la investigación incluye la medida de un conjunto de constructos psicológicos que hacen referencia a la empatía, inestabilidad emocional, mecanismos de afrontamiento y conducta agresiva.

\section{Análisis de datos}

Se realizaron análisis de varianza para establecer las diferencias entre grupos en las variables evaluadas. Para poner a prueba los tres modelos teóricos hipotetizados, según el tipo de afrontamiento analizado en cada grupo de edad y evaluar si presentan un buen ajuste a los datos empíricos, se utilizaron ecuaciones estructurales (Kline, 1998) que fueron analizadas a través del programa AMOS Graphics 16.0 (Arbuckle, 2006). Para evaluar el ajuste de cada uno de los modelos se emplearon los siguientes índices: $\chi^{2}, \chi^{2} / g l$, GFI (Goodness of Fit Index), AGFI (Adjusted Goodnessof Fit Index) y el Índice de Ajuste Normativo Bentler-Bonet ([NFI], BentlerBonett Normed Fit Index). Por último, para estimar el grado de error del modelo se calcularon el RMR (Root Mean Square Residual) y el RMSEA (Root Mean Standar Error).

\section{Resultados}

\section{Diferencias de edad en las variables evaluadas}

Se ha categorizado la variable edad en dos grupos (1213 años y 14-15 años). Los análisis de varianza realizados muestran que no hay diferencias significativas en Empatía $(F(1,1554)=0.541 ; p=0.462)$ entre los dos grupos de edad. Sí se constatan dichas diferencias en Agresividad $(F(1,1554)=4.085 ; p<0.05)$ y en Inestabilidad Emocional $(F(1,1554)=6.189$; $p<0.05)$, de manera que son los adolescentes de mayor edad (14-15 años) los que más alto han puntuado en agresividad física y verbal y en inestabilidad emocional. Además, hay diferencias significativas en las estrategias de Invertir en amigos íntimos $(F(1,1335)=8.824 ; p<0.01)$ y en Reducción de la tensión $(F(1,1335)=8.554 ; p<0.05)$, siendo en ambos casos los adolescentes mayores (14-15 años) los que utilizan en mayor medida dichas estrategias de afrontamiento. Al realizar el análisis de varianza, agrupando las diferentes estrategias en los tres estilos básicos de afrontamiento, los resultados no señalan diferencias estadísticamente significativas.

Por otro lado, independientemente de la edad, los adolescentes utilizan en mayor medida las siguientes estrategias: Buscar diversiones relajantes $(M=68.55)$, Distracción física $(M=67.48)$, Preocuparse $(M=65.45)$, Buscar Pertenencia $(M=65.12)$, Esforzarse y tener éxito $(M=65.07)$, Fijarse en lo positivo $(M=63.54)$, Concentrarse en resolver el problema $(M=63.12)$, Invertir en amigos íntimos $(M=62.85)$, Búsqueda de apoyo social $(M=62.04)$. Mientras que emplean raras veces: Buscar apoyo espiritual $(M=46.15)$, Ignorar el problema $(M=44.79)$, Falta de afrontamiento $(M=43.9)$, Acción social $(M=42.85)$ y Reducción de la tensión $(M=39.37)$. Las estrategias menos utilizadas por la muestra de adolescentes, pertenecen al estilo de afrontamiento improductivo. Estos resultados son muy similares a los obtenidos en otros estudios (Gómez-Fraguela et al., 2006; Pereña \& Seisdedos, 1997).

\section{Relación entre estilos de afrontamiento $y$ conducta agresiva}

Siguiendo con el objetivo general del estudio, se ha analizado la utilización de las diferentes estrategias y estilos de afrontamiento en función del nivel de agresividad de los adolescentes, según sean más o menos agresivos. Para esto, se han constituido dos grupos extremos de baja y alta agresividad, agrupando a los sujetos que han alcanzado una puntuación por debajo de la media y una desviación típica en agresividad física y verbal $(N=230)$ en el grupo de baja agresividad y aquellos que han alcanzado una puntuación por encima de la media y una desviación típica en el grupo de alta agresi$\operatorname{vidad}(N=194)$. El ANOVA realizado indica que los adolescentes que puntúan más bajo en agresividad utilizan en mayor medida mecanismos de afrontamiento centrados en Resolver el problema $(F(1,422)=9.770, p<0.01)$, en Esforzarse y tener éxito $(F(1,422)=25.764, p<0.01)$, en buscar apoyo social $(F(1,422)=14.272, p<0.01)$, preocuparse $(F(1,422)=4.249, p<0.05)$ y Fijarse en 
lo positivo $(F(1,422)=5.857, p<0.05)$, estrategias que forman parte de dos estilos de afrontamiento: el dirigido a la resolución del problema y el que se apoya en la relación con los demás. Por el contrario, la alta agresividad se relaciona con Falta de afrontamiento $(F(1,422)=33.962, p<0.01)$,
Ignorar el problema $(F(1,422)=15.654, p<0.01)$ Reservarlo para sí $(F(1,422)=7.292, \mathrm{p}<0.01)$, o simplemente Preocuparse por reducir la tensión $(\mathrm{F}(1,422)=38.485, \mathrm{p}<0.01)$, se trata en este caso de estrategias que describen el afrontamiento improductivo.

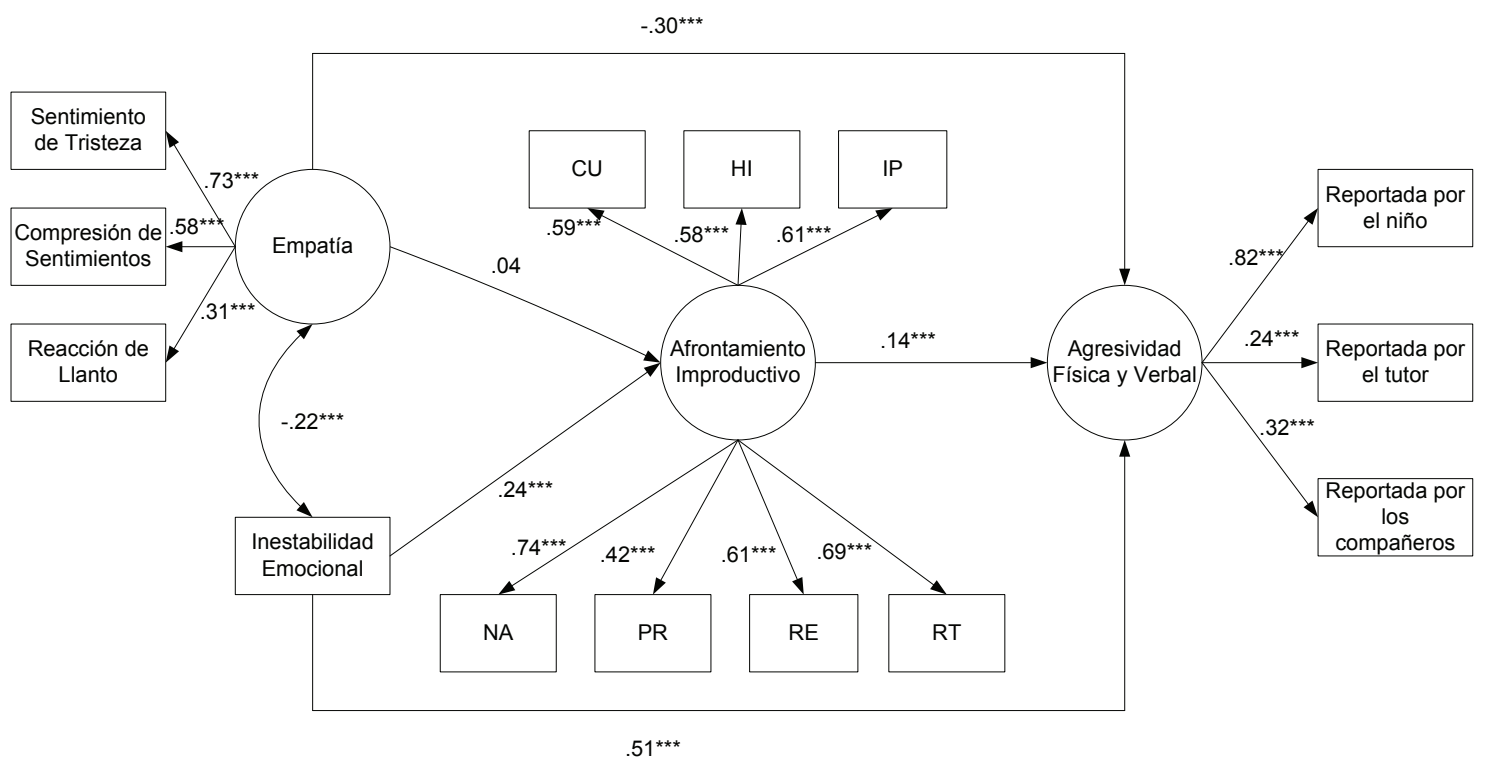

Figura 1. Empatía, Inestabilidad emocional, Afrontamiento improductivo y Conducta agresiva en niñez tardía.

$\mathrm{N}=1.074$ (12 y 13 años)

Fuente: elaboración propia.

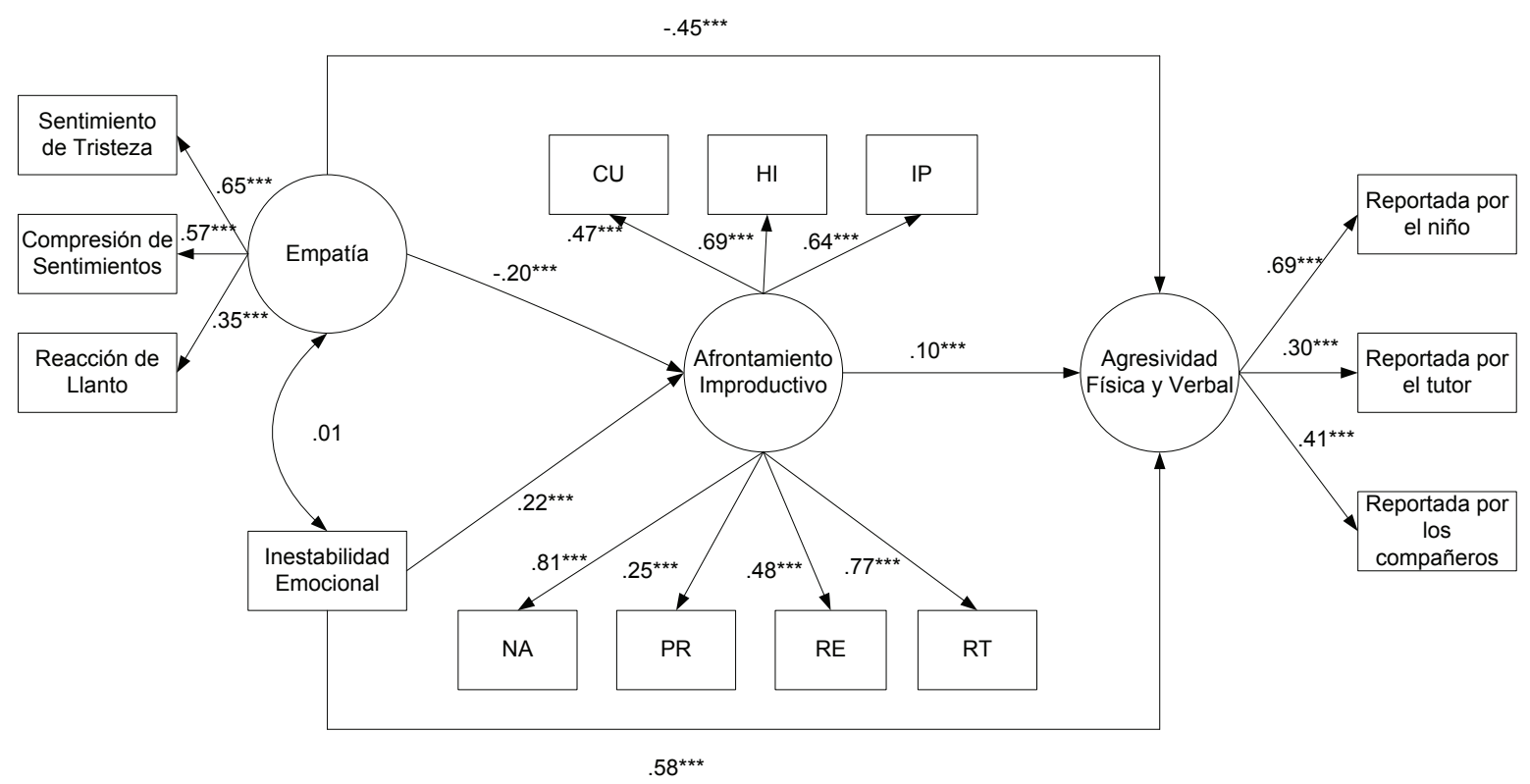

Figura 2. Empatía, Inestabilidad emocional, Afrontamiento improductivo y Conducta agresiva en la adolescencia temprana.

$\mathrm{N}=482$ (14 y 15 años)

Fuente: elaboración propia. 


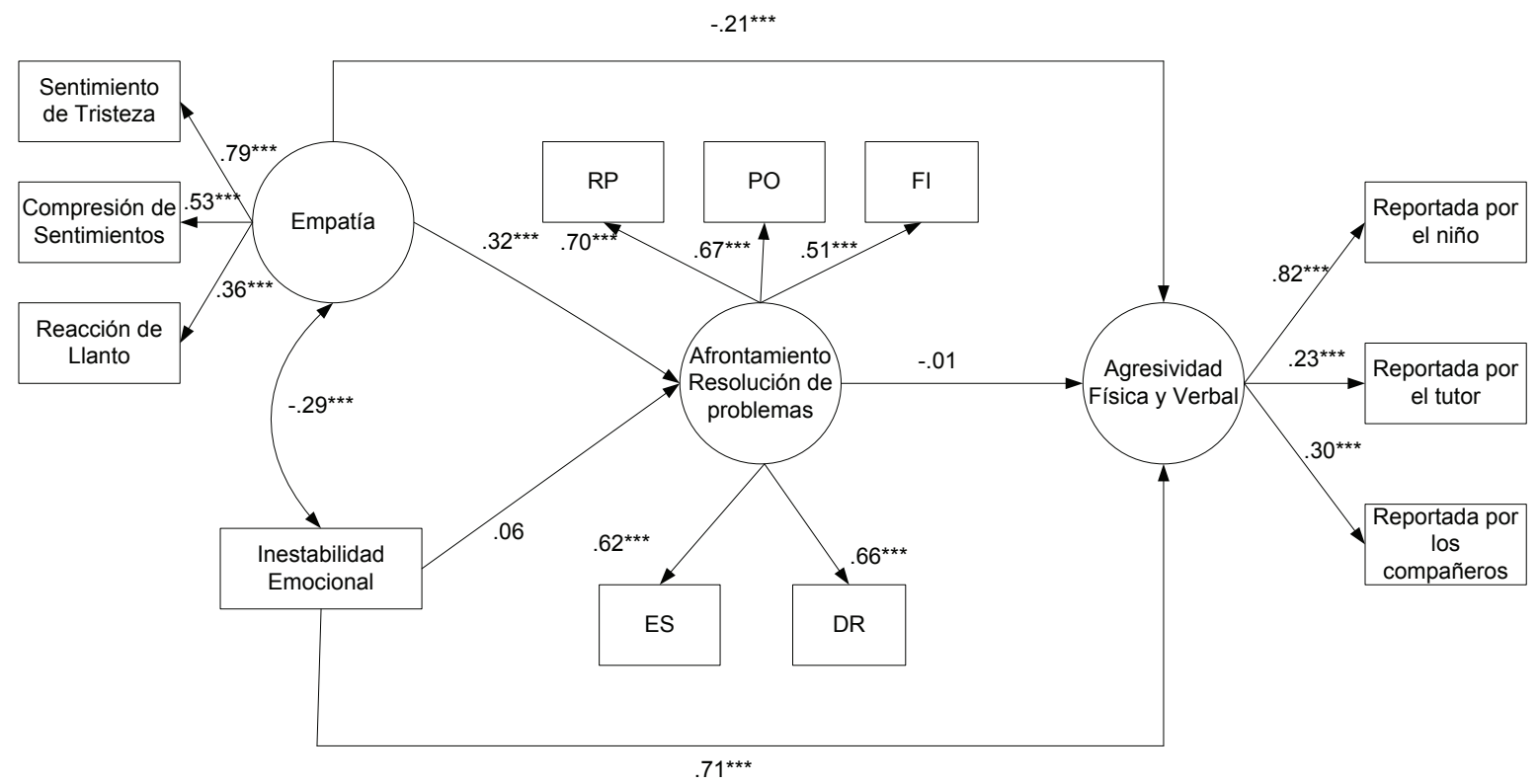

Figura 3. Empatía, Inestabilidad emocional, Afrontamiento resolución de problemas y Conducta agresiva en niñez tardía. $\mathrm{N}=1.074$ (12 y 13 años)

Fuente: elaboración propia.

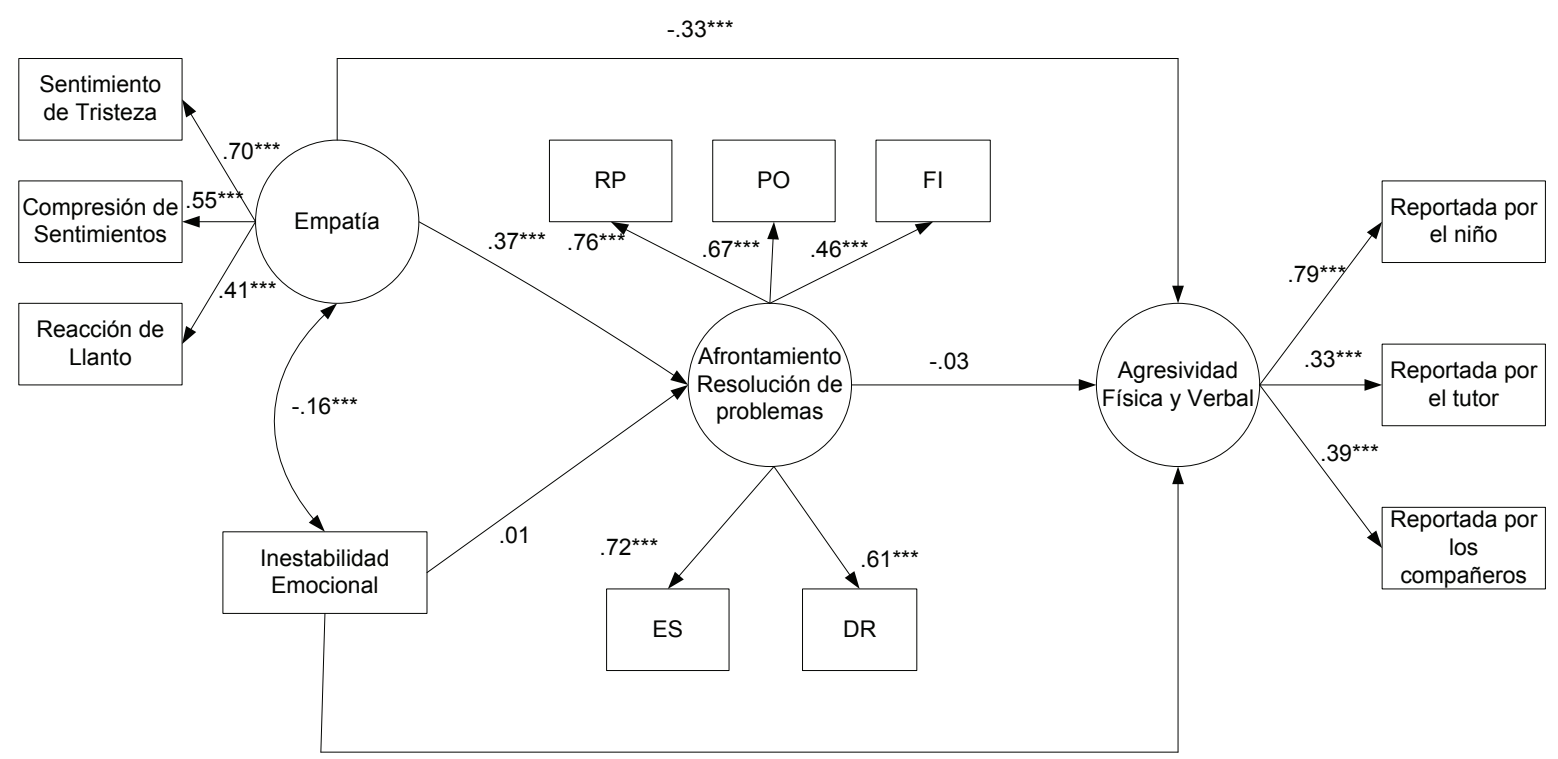

$65^{\star * *}$

Figura 4. Empatía, Inestabilidad emocional, Afrontamiento resolución de problemas y Conducta agresiva en adolescencia temprana.

$\mathrm{N}=482(14$ y 15 años $)$

Fuente: elaboración propia. 


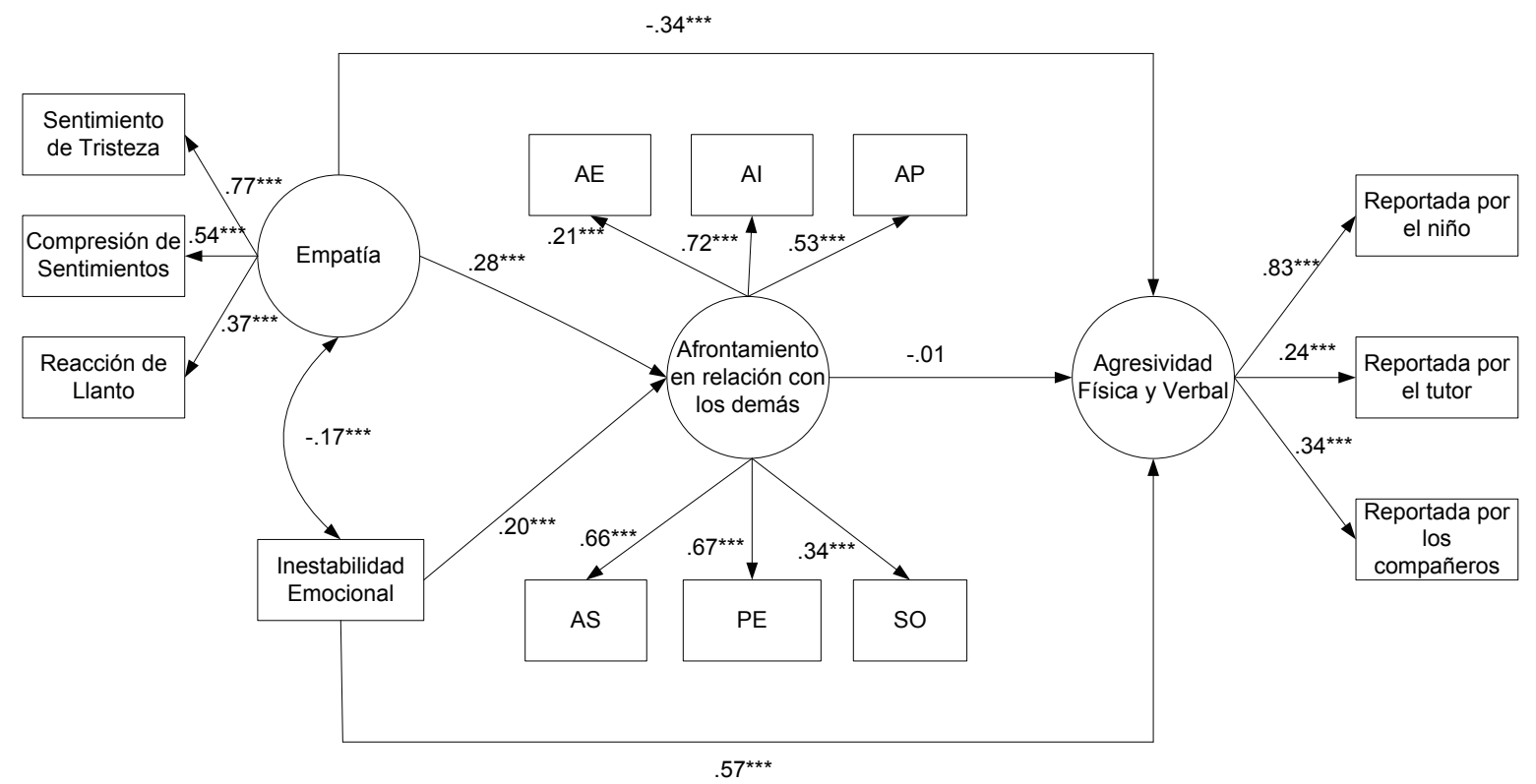

Figura 5. Empatía, Inestabilidad emocional, Afrontamiento en relación con los demás y Conducta agresiva en la niñez tardía.

$\mathrm{N}=1.074$ (12 y 13 años)

Fuente: elaboración propia.

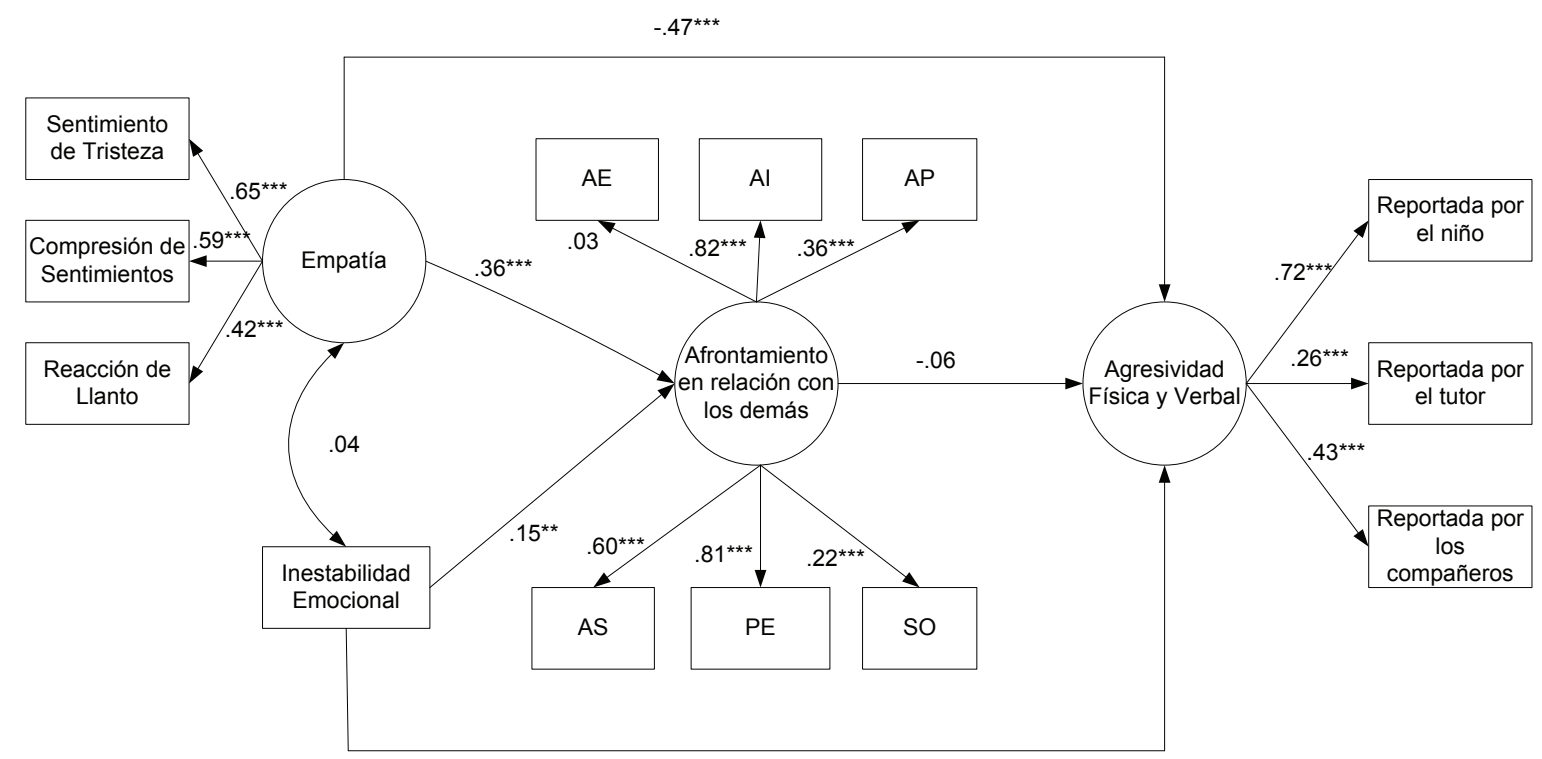

$.65^{* * *}$

Figura 6. Empatía, Inestabilidad emocional, afrontamiento en relación con los demás y conducta agresiva en adolescencia temprana.

$\mathrm{N}=482$ (14 y 15 años)

Fuente: elaboración propia. 


\section{Modelos de Ecuaciones Estructurales}

En relación con las hipótesis 2 y 3 , se presentan a continuación los tres modelos teóricos hipotetizados, según el tipo de afrontamiento analizado en cada grupo de edad, la empatía, la inestabilidad emocional y su relación con la agresividad.

\section{Modelo 1. Empatía, Inestabilidad emocional,} Afrontamiento improductivo y Conducta agresiva en la niñez tardía y en la adolescencia temprana

En la Figura 1 se presenta el modelo híbrido de ecuaciones estructurales con sus respectivos coeficientes de path para la muestra de niños y en la Figura 2 para la de los adolescentes. Se obtuvieron un $\chi^{2}(72)=326.62, p<0.001, \chi^{2} / g 1=4.54$, $\mathrm{GFI}=0.93$; AGFI $=0.90$, para la muestra los niños y $\chi^{2}(72)=274, p<0.001, \chi^{2} / g 1=3.8$, $\mathrm{GFI}=0.91 ; \mathrm{AGFI}=0.87$, para la muestra de adolescentes. Ambos modelos mostraron un buen ajuste a los datos empíricos.

Seguidamente, se calcularon los índices de error, obteniéndose para la muestra de niños $\mathrm{RMR}=0.04$ y $\mathrm{RMSEA}=0.06$ y para la muestra de adolescentes $\mathrm{RMR}=0.05$ y $\mathrm{RMSEA}=0.07$.

\section{Modelo 2. Empatía, Inestabilidad emocional,} Afrontamiento centrado en la resolución de problemas y Conducta agresiva en la niñez tardía y en la adolescencia temprana

Las Figuras 3 y 4 muestran los coeficientes de path para la muestra de niños y adolescentes respectivamente, para el segundo modelo estudiado. Ambos modelos obtuvieron un buen ajuste $\chi^{2}(49)=207.11, p<0.001, \chi^{2} / g 1=4.23$, $\mathrm{GFI}=0.97$ AGFI $=0.95, \mathrm{RMR}=0.09 \mathrm{y}$ RMSEA $=0.05$, para la muestra de niños $\mathrm{y}$ $\chi^{2}(49)=118, p<0.001, \chi^{2} / \mathrm{gl}=3.02, \mathrm{GFI}=0.96$; $\mathrm{AGFI}=0.94, \mathrm{RMR}=0.11$ y RMSEA $=0.05$, para la muestra de adolescentes. Téngase en cuenta que el error se encuentra algo aumentado en el análisis de los modelos de ambas muestras.
Modelo 3. Empatía, Inestabilidad

emocional, Afrontamiento relación con

los demás y Conducta agresiva en la niñez

tardía y en la adolescencia temprana

Las Figuras 5 y 6 muestran los coeficientes de path para la muestra de niños y adolescentes, respectivamente, para el tercer modelo estudiado. Ambos modelos obtuvieron un ajuste aceptable. El modelo analizado con la muestra de niños obtuvo $\chi^{2}(60)=727.9, p<0.001, \chi^{2} / g l=12.13, \mathrm{GFI}=0.90 ;$ $\mathrm{AGFI}=0.84, \mathrm{RMR}=0.04$ y $\mathrm{RMSEA}=0.10$; el modelo analizado con la muestra de adolescentes $\chi^{2}(60)=458.7, p<0.001, \chi^{2} / g l=7.6$, GFI $=0.86$; AGFI $=0.79, \mathrm{RMR}=0.05$ y $\mathrm{RMSEA}=0.12 . \mathrm{El}$ modelo, en el análisis de ambas muestras, obtuvo un valor $\chi^{2} / g l$ y de error aumentados para los niveles esperados.

\section{Discusión}

Los resultados de este estudio muestran diferencias significativas en función de la edad en algunas de las variables evaluadas. Destaca en primer lugar el aumento de la agresividad física y verbal y la inestabilidad emocional entre los 12 y los 15 años, datos que son coherentes con otros estudios realizados en nuestra población (Carlo et al., 2010; López \& De la Caba, 2011; Mestre et al., 2010; Mora-Merchán, 2006; Samper, Tur, Mestre \& Cortés, 2008). Resultados que ponen de relieve la necesidad de intervenir durante la infancia con el objetivo de prevenir el incremento de la agresividad en el inicio de la adolescencia.

La edad no parece marcar grandes diferencias en los mecanismos de afrontamiento utilizados, aunque se constata que los adolescentes entre 12 y 15 años utilizan en mayor medida como estrategias de afrontamiento el buscar diversiones relajantes, la distracción física, el esforzarse y tener éxito y el concentrarse en resolver el problema, todas ellas forman parte del estilo de afrontamiento Dirigido a la resolución del problema. Además, también destaca la utilización de algunas estrategias que definen el afrontamiento centrado en la relación con los demás, tales como invertir en amigos íntimos, buscar 
apoyo social, o buscar ayuda profesional, mientras que se utilizan en menor medida estrategias como buscar apoyo espiritual o ignorar el problema. Otros estudios en diferentes poblaciones han concluido un mayor peso de actitudes de afrontamiento positivas en la adolescencia frente a las negativas o pasivas (Gómez-Fraguela et al., 2006; López \& De la Caba, 2011; Veloso-Besio, Caqueo-Arancibia, CaqueoUrizar, Muñoz-Sánchez \& Villegas-Abarzúa, 2010).

En relación con la primera hipótesis, el ANOVA realizado entre los sujetos con baja y alta agresividad apoya esta hipótesis, al establecer diferencias significativas entre los estilos de afrontamiento que utilizan los dos grupos extremos en relación con la agresividad física y verbal, siendo los sujetos menos agresivos los que utilizan en mayor medida, ante situaciones conflictivas o que producen tensión, estrategias propias de un estilo de afrontamiento centrado en la resolución del problema y en la relación con los demás, mientras que el afrontamiento improductivo es más frecuente entre los adolescentes clasificados en el grupo de mayor agresividad. Por tanto, se confirma la relación entre el afrontamiento y la agresividad y, por tanto, el carácter protector que los estilos de afrontamiento centrados en la resolución del problema y en la relación con los demás ejercen frente a la agresividad, mientras que el afrontamiento improductivo ejerce el efecto contrario (Arán-Filippetti \& Richaud, 2011; Mora-Merchán, 2006; Quintana, Montgomery \& Malaver, 2009). Por tanto, la investigación en esta línea aporta resultados que apoyan la relación entre el afrontamiento y el ajuste psicosocial de los adolescentes (Clarke, 2006).

Los Modelos de Ecuaciones Estructurales formulados según el tipo de afrontamiento analizado en cada grupo de edad (12-13 años y 14-15 años), la empatía, la inestabilidad emocional y su relación con la agresividad, permiten confirmar la segunda y tercera hipótesis.

En el primer modelo se encuadran diferencias entre las edades; los más jóvenes presentan una correlación negativa y significativa entre empatía e inestabilidad emocional, cosa que no ocurre en los más mayores. Por otra parte, en los más pequeños la empatía no se relaciona con el afrontamiento improductivo, mientras sí lo hace en los más grandes. Es decir, en los adolescentes la falta de empatía favorece un tipo de afrontamiento improductivo. La inestabilidad emocional se relaciona positivamente con el afrontamiento improductivo y este con la agresividad en ambas edades.

Según el segundo modelo, tanto los de menor edad (12-13 años) como los adolescentes (14-15 años) presentaron una relación positiva de la empatía con el afrontamiento centrado en la resolución de problemas, que a su vez no tiene relación con la agresividad; por su parte, el efecto de la inestabilidad emocional desaparece. Según este modelo, la empatía favorece el afrontamiento centrado en la resolución de problemas en los dos grupos de edad. Estos resultados señalan la importancia de desarrollar la empatía a lo largo de la infancia y la adolescencia.

En el tercer modelo, en ambas edades tanto la empatía como la inestabilidad emocional se relacionan positivamente con el afrontamiento en relación con los demás, pero este último no se relaciona con la agresividad. Estos resultados hacen pensar en un afrontamiento de búsqueda de apoyo en los demás pero no instrumental. De alguna manera, cuando los niños experimentan esa inestabilidad acuden en mayor medida en búsqueda de apoyo de los adultos, pares, etc. Es un patrón que se encuentra en los dos grupos de edad.

Los tres modelos permiten confirmar la tercera hipótesis: la empatía y la inestabilidad emocional en la niñez tardía y en la adolescencia temprana tienen un efecto diferencial según los diferentes tipos de afrontamiento (improductivo, resolución de problemas y relación con los demás). En resumen, la empatía facilita el afrontamiento centrado en la resolución de problemas, mientras que la inestabilidad emocional favorece el afrontamiento improductivo, también en el grupo de adolescentes de mayor edad la falta de empatía guarda relación con un estilo de afrontamiento más improductivo. Tanto la empatía como la inestabilidad emocional favorecen el afrontamiento centrado en la relación con los demás.

Además, en todos los modelos el efecto directo de la inestabilidad emocional y la empatía sobre la 
agresividad es significativo, siempre positivo en el primer caso pero negativo en el segundo. Estos resultados confirman la segunda hipótesis e indican la importancia de la empatía como una emoción orientada al otro, que incluye la comprensión y la expresión de sentimientos, en la inhibición de la agresividad. Por el contrario, la inestabilidad emocional y la falta de autocontrol en situaciones sociales como resultado de la escasa capacidad para frenar la impulsividad y la emocionalidad, correlaciona negativamente con la empatía y tiene un efecto directo y positivo sobre la agresividad. Estos resultados son coherentes con otros estudios realizados en la infancia y la adolescencia que concluyen el importante papel de la empatía, en su dimensión cognitiva y emocional, en la disposición prosocial y, por el contrario, el efecto de la impulsividad y la falta de autocontrol en la agresividad y desajuste social de los adolescentes (Caprara \& Pastorelli, 1993; Carlo et al., 2010; Del Barrio et al., 2009).

El presente estudio tiene algunas limitaciones. La naturaleza transversal del mismo no permite realizar inferencias de causalidad ni observar cambios entre las variables evaluadas a lo largo del tiempo, por lo que se propone desarrollar estudios longitudinales sobre emociones, estilos de afrontamiento, conducta agresiva y conducta prosocial. Sin embargo, el presente estudio, realizado sobre una muestra amplia de sujetos y representativa de la población escolarizada, aporta resultados nuevos sobre procesos psicológicos que guardan relación con la conducta agresiva. Si bien la investigación sobre empatía y conducta prosocial se ha incrementado en los últimos años, no se han estudiado en la misma medida los estilos de afrontamiento y su relación con las emociones y la conducta del sujeto. Las diferencias obtenidas entre los estilos de afrontamiento en relación con las emociones y la conducta agresiva deben ser tenidas en cuenta para la prevención de la agresividad y el desarrollo de conductas adaptadas en la adolescencia. La carencia de estrategias de afrontamiento adecuadas junto con la falta de empatía o una alta inestabilidad emocional, dificulta la capacidad de los adolescentes para afrontar situaciones conflictivas y resolverlas de manera adaptativa.
Finalmente, los resultados obtenidos plantean la necesidad de intervenir precozmente a través de programas que desarrollen la empatía, los mecanismos de afrontamiento, el autocontrol y en definitiva la capacidad para afrontar situaciones problemáticas y de riesgo (Arán- Filippetti \& Richaud, 2011; Garaigordobil, 2009a; Mestre, Tur, Samper \& Malonda, 2011).

\section{Referencias}

Arán- Filippetti , V. \& Richaud de Minzi, M. C.(2011). Efectos de un programa de intervención para aumentar la reflexividad y la planificación en un ámbito escolar de alto riesgo por pobreza. Universitas Psychologica, 10(2), 341-354.

Arbuckle J. (2006). Amos 7.0 User's Guide. Spring House, PA: Amos Development Corporation.

Bermúdez, M. P., Teva, I. \& Buela-Casal, G. (2009). Influencia de variables sociodemográficas sobre los estilos de afrontamiento, el estrés social y la búsqueda de sensaciones sexuales en adolescentes. Psicothema, 21(2), 220-226.

Bermúdez, M. P., Teva, I. \& Sánchez, A. (2003). Análisis de la relación entre inteligencia emocional, estabilidad emocional y bienestar psicológico. Universitas Psychologica, 2(1), 27-32.

Bryant, B. K. (1982). An index of empathy for children and adolescents. Child Development, 53, 413-425.

Caprara, G. V. \& Pastorelli, C. (1993). Early emotional instability, prosocial behavior, and aggression: Some methodological aspects. European Journal of Personality, 7(1), 19-36.

Caprara, G. V., Barbanelli, C. L., Pastorelli, C., Bandura, A. \& Zimargdo, Ph. G. (2000). Prosocial foundations of children's academic achievement. Psychological Science, 11(4), 302-306.

Carlo, G., Mestre, M. V., Samper, P., Tur, A. \& Armenta, B. E. (2010). Feelings or cognitions? Moral cognitions and emotions as longitudinal predictors of prosocial and aggressive behaviors. Personality and Individual Differences, 48(8), 865-962.

Chaux, E. (2003). Agresión reactiva, agresión instrumental y el ciclo de la violencia. Revista de Estudios Sociales, 15, 47-58. 
Clarke, A. T. (2006). Coping with interpersonal stress and psychosocial health among children and adolescents: A meta-analysis. Journal of Youth and Adolescence, 35(1), 11-24.

Del Barrio, V., Carrasco, M. A., Rodríguez, M. A. \& Gordillo, R. (2009). Prevención de la agresión en la infancia y la adolescencia. International Journal of Psychology and Psychological Therapy, 9(1), 101-107.

Del Barrio, V., Moreno, C. \& López, R. (2001). Evaluación de la agresión e inestabilidad emocional en niños españoles y su relación con la depresión. Clínica y Salud, 13(1), 33-50.

Dovidio, J. F. \& Penner, L. A. (2004). Helping and altruism. En M. B. Brewer \& M. Hewstone (Eds.), Emotion and motivation. Perspectives on social psychology (pp. 247-280). Malden, MA: Blackwell Publishers.

Farrington, D. P. (2004). Conduct disorder, aggression, and delinquency. In R. M. Lerner \& L. Steinberg (Eds.), Handbook of adolescent psychology (2nd. ed., pp. 627-664). Hoboken, NJ: Wiley.

Frydenberg, E. \& Lewis, R. (1995). ACS. Escalas de afrontamiento para Adolescentes. Madrid: TEA.

Garaigordobil, M. (2009a). Intervención socioeducativa para promover la conducta prosocial y prevenir la violencia. Organización y Gestión Educativa, 3, 31-32.

Garaigordobil, M. (2009b). Factores familiares, escolares y personales que favorecen la convivencia. IHITZA, 29, 25.

Gómez-Fraguela, J. A., Luengo, A., Romero, E., Villar, P. \& Sobral, J. (2006). Estrategias de afrontamiento en el inicio de la adolescencia y su relación con el consumo de drogas y la conducta problemática. International Journal of Clinical and Health Psycho$\log y, 6(3), 581-597$.

Ison-Zintilini, M. S. \& Morelato, G. S. (2008). Habilidades socio-cognitivas en niños con conductas disruptivas y víctimas de maltrato. Universitas Psychologica, 7(2), 357-367.

Kline, R. B. (1998). Principles and practices of structural equation modeling. New York: Guilford.

López, R. \& De la Caba, M. A. (2011). Estrategias de afrontamiento ante el maltrato escolar en estudiantes de primaria y secundaria. Aula Abierta, 39(1), 59-68.
Mestre, M. V., Pérez-Delgado, E., Frías, D. \& Samper, P. (1999). Instrumentos de evaluación de la empatía. En E. Pérez-Delgado \& V. Mestre (Eds.), Psicología moral y crecimiento personal (pp. 181-190). Barcelona. Ariel.

Mestre, V, Samper, P. \& Frías, M. D. (2004). Personalidad y contexto familiar como factores predictores de la disposición prosocial y antisocial de los adolescentes. Revista Latinoamericana de Psicología, 36(3), 445-457.

Mestre, V., Samper, P. \& Frías, M. D. (2002). Procesos cognitivos y emocionales predictores de la conducta prosocial y agresiva: la empatía como factor modulador. Psicothema, 14(2), 227-232.

Mestre, V., Tur, A., Samper, P. \& Latorre, A. (2010). Relaciones entre la inestabilidad emocional y la agresión. La acción de los estilos de crianza. Ansiedad y Estrés, 16(1), 33-45.

Mestre, V., Tur, A., Samper, P. \& Malonda, E. (2011). Programa de educación de las emocions: La ConVivencia. Valencia: Tirant Lo Blanc.

Mora-Merchán, J. A. (2006). Las estrategias de afrontamiento, imediadoras de los efectos a largo plazo de las víctimas de bullying? Anuario de Psicología Clínica y de la Salud, 2, 15-26.

Páez, D. (1993). Salud, expresión y represión social de las emociones. Valencia: Promolibro.

Pereña, J. \& Seisdedos, N. (1997). ACS. Escalas de afrontamiento para Adolescentes. Madrid: TEA.

Perona, A. \& Galán, S. (2001). Algunas aportaciones críticas en torno a la búsqueda de un marco teórico del afrontamiento en la psicosis. Psicothema, 13(4), 563-570.

Quintana, A., Montgomery, W. \& Malaver, C. (2009). Modos de afrontamiento y conducta resiliente en adolescentes espectadores de violencia entre pares. Revista de Investigación en Psicología, 12(1), 153-171.

Richaud, C. (2006). Evaluación del afrontamiento en niños de 8 a 12 años. Revista Mexicana de Psicología, 23(2), 196-201.

Samper, P., Aparici, G. \& Mestre, V. (2006). La agresividad auto y heteroevaluada: variables implicadas. Acción Psicológica, 4(2), 155-168.

Samper, P., Tur, A., Mestre, V. \& Cortés, M. T. (2008). Agresividad y afrontamiento en la adolescencia. Una perspectiva intercultural. International Jour- 
nal of Psychology and Psychological Therapy, 8(3), 431-440.

Seligman, M. E. P. \& Csikszentimihalyi, M. (2000). Positive psychology: An introduction. American Psychologist, 55(1), 5-14.

Tur, A. M., Mestre, V. \& Del Barrio, V. (2004). Los problemas de conducta exteriorizados e interiorizados en la adolescencia: relaciones con los hábitos de crianza y con el temperamento. Acción Psicológica, 3(3), 207-221.
Vaish, A., Carpenter, M. \& Tomasello, M. (2009). Sympathy through affective perspective taking and its relation to prosocial behavior in toddlers. Developmental Psychology, 45(2), 534-543.

Veloso-Besio, C., Caqueo-Arancibia, W., Caqueo-Urizar, A., Muñoz-Sánchez, Z. \& Villegas-Abarzúa, F. (2010). Estrategias de afrontamiento en adolescentes. Revista de Psicología, 22 (1), 23-34. 
